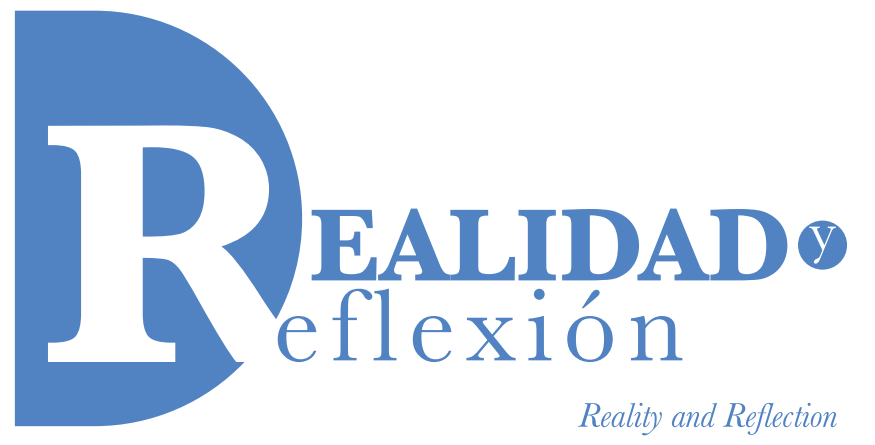

ISSN 1992-6510

e-ISSN 2520-9299

Año 18, N 47, San Salvador, El Salvador, Centroamérica. Revista Semestral Enero-Junio 2018

YEAR 18, N 47, SAN SALVADOR, EL SALVADOR, CENTRAL AMERICA. SEMESTRAL JOURNAL JANUARY-JUNE 2018

\title{
Estudio de materiales de bajo coste para la mejora de la desalinización solar de agua de mar
}

\author{
Study of low cost materials for the enhancement of \\ solar seawater desalination
}

\author{
Rainer Christoph \\ Master in Physical Chemistry, University of Bern \\ Ángel Hernández \\ Ingeniero en Sistemas Informáticos, Universidad de El Salvador \\ Romeo Muñoz \\ Estudiante egresado Ingeniería en Sistemas, Universidad de E1 Salvador \\ Jonathan Ventura \\ Estudiante de $5^{\circ}$ año de Ingeniería Industrial, Universidad Francisco Gavidia \\ Wolfgang Büscher \\ Master in Mechanical Engineering, Technical University Munich, Germany \\ Recibido: 19 de enero de 2018 \\ Aprobado: 18 de mayo de 2018 \\ DOI: http://dx.doi.org/10.5377/ryr.v0i47.6275
}

Todos los autores trabajan en el Laboratorio de Nanotecnología de la Universidad Francisco Gavidia

\section{RESUMEN}

La evaporación de agua altamente eficiente bajo iluminación solar es de importancia clave para el rendimiento de los alambiques de desalinización de agua de mar. La eficiencia de la generación de vapor térmico aumenta notablemente a través del aislamiento térmico de las capas superficiales de agua irradiada del agua subyacente. Estudios recientes han demostrado que el uso de nanomateriales con propiedades plasmónicas superficiales da lugar a aumentos adicionales de la eficiencia térmica. Presentamos los resultados de los experimentos de evaporación de agua in situ realizados en condiciones ambientales normales. El uso de estructuras flotantes de capas de sándwich (SLS), puede incrementar las tasas de evaporación de agua de hasta un 43\% en comparación con las tasas obtenidas para superficies de agua lisa con un bulto expuesto a la luz subyacente. La importancia del transporte acuático para la superficie SLS expuesta a la luz, y por lo tanto el diseño SLS es crucial, como demostramos con un conjunto adicional de experimentos. La eficiencia de evaporación aumenta cuando en la superficie iluminada SLS hay capas de polvo impregnadas en agua de absorbentes de luz fuertes. Los resultados comparativos obtenidos con las capas de grafito en polvo 
húmedo y los compuestos de rocas ígneas muestran que, en las mismas condiciones experimentales, la tasa de evaporación del agua puede aumentar hasta un 470\%, en comparación con las superficies de agua desnudas.

Palabras clave: desalinización, destilador solar, evaporación, grafito, nanomateriales, roca ígnea.

\section{ABSTRACT}

Highly efficient water evaporation under solar illumination is of key importance for the performance of seawater desalination stills. Thermal vapor generation efficiency increases markedly through thermal insulation of the irradiated water surface layers from the underlying bulk water. Recent studies have shown that the use of nanomaterials with plasmonic surface properties lead to additional thermal efficiency boosts. We present results of in situ water evaporation experiments performed under normal ambient conditions. The use of floating sandwich-layered structures (SLS), can increment water evaporation rates of up to $43 \%$ when compared to the rates obtained for plain water surfaces with an underlying light-exposed bulk. The importance of water transport to the light-exposed SLS surface, and hence the SLS design is crucial, as we demonstrate with an additional set of experiments. Evaporation efficiency increases when water impregnated powder layers of strong light absorbers are present at the illuminated SLS surface. Comparative results obtained with wet powder layers of graphite and igneous rock compounds show that, under same experimental conditions, the water evaporation rate can be boosted by as much as $470 \%$ compared to bare water surfaces.

Keywords: desalination, evaporation, graphite, igneous rock, nanomaterials, solar stills.

\section{Introducción}

El agua es clave para el desarrollo sostenible. Los recursos hídricos y la gama de servicios que proporcionan, sustentan la reducción de la pobreza, el crecimiento económico y la sostenibilidad ambiental. Desde la seguridad alimentaria y energética hasta la salud humana y ambiental, el agua contribuye a mejorar el bienestar social y el crecimiento inclusivo, afectando los medios de subsistencia de miles de millones de personas (UN-WWAP, 2015).

Aproximadamente e1 97\% del agua de la Tierra es agua salada en los océanos y sólo el 3\% (cerca de 35 millones de $\mathrm{km} 3$ ) es agua dulce contenida en los polos (en forma de hielo), aguas subterráneas, lagos y ríos, que abastecen la mayoría de las necesidades humanas y animales (Fig. n. ${ }^{\circ} 1$ ). Muchos de estos sistemas de agua dulce están directamente amenazados por el crecimiento de la población humana, la agricultura, la industrialización y la contaminación. También se verán aún más afectados por el cambio climático antropogénico (NASA Climate Change Webpage, 2017), (Haddeland, et al., 2014) que tiene el potencial de perturbar los patrones de disponibilidad de agua que han motivado decisiones con implicaciones a largo plazo, tales como la ubicación de las ciudades, la estructura urbana, la ubicación de los embalses y el diseño del sistema hídrico (Bates et al., 2008). (Ver Figura n. ${ }^{\circ}$ 1)

En la actualidad, más de un tercio de la población mundial vive en países afectados por el estrés hídrico y para 2025 se prevé que esta cifra aumentará a casi dos tercios (Service, 2006). La escasez de agua es uno de los desafíos globales más graves de nuestro tiempo, a pesar de ser uno de los recursos más abundantes de la tierra, cubriendo las tres cuartas partes de la superficie del planeta. Incluso las regiones tradicionalmente consideradas ricas en agua tienen dificultades para proveer suficiente agua dulce (Supply et al., 2011). La contaminación y el aumento de los costos asociados con el tratamiento y la distribución de nuevas fuentes 


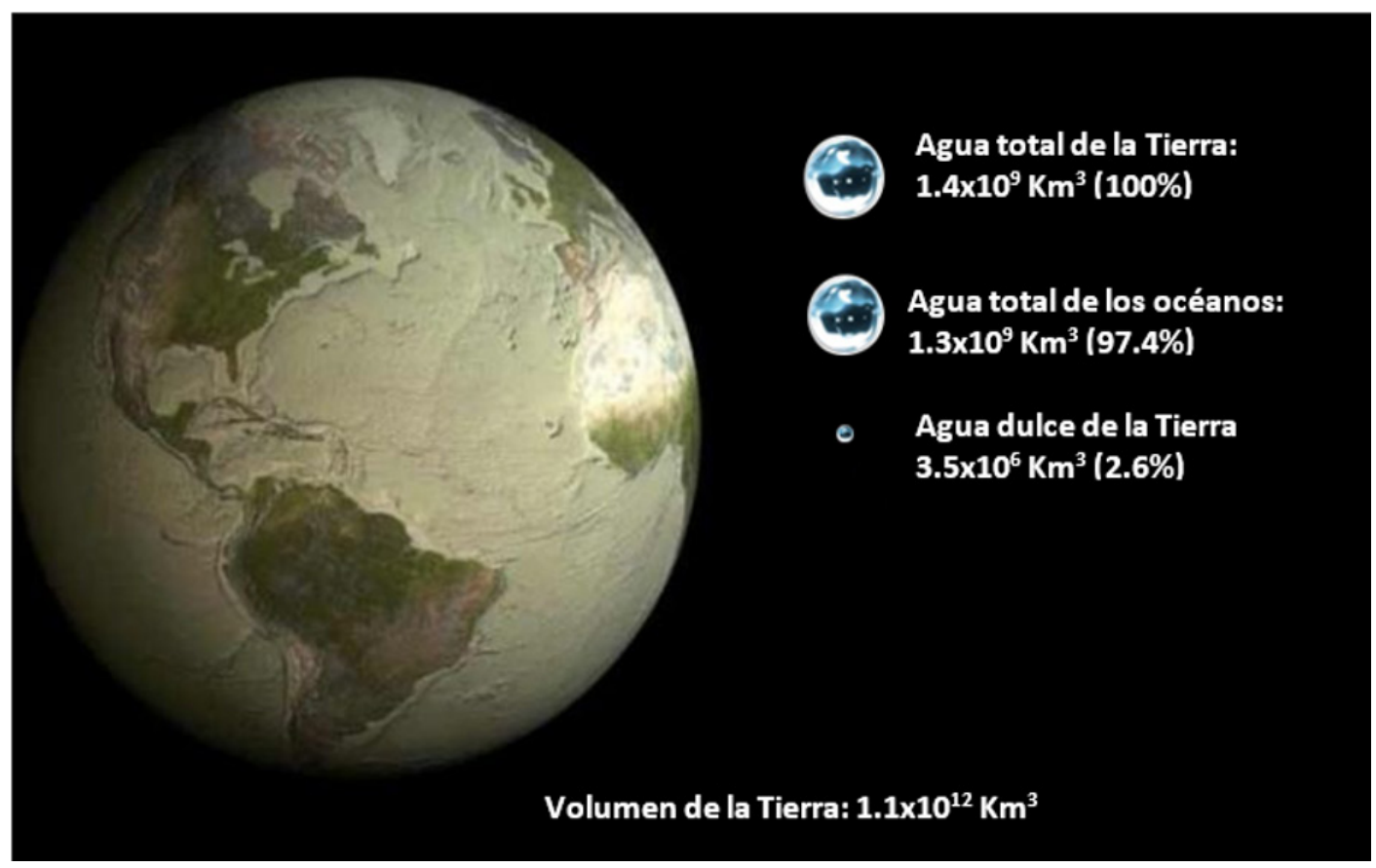

Fig. 1. Distribución de aguas superficiales en el planeta Tierra. El agua dulce representa menos del $3 \%$ del recurso hídrico total del planeta. Casi el 70\% del agua dulce se congela en glaciares, nieve permanente y permafrost. El 30\% de toda el agua dulce es subterránea, la mayoría en acuíferos profundos y de difícil acceso. Los lagos y ríos juntos contienen sólo un poco más del 0,25\% de toda el agua dulce, o menos del 0,01\% del agua total (Gleick, 1993).

de agua son algunos de los principales problemas a los que se enfrentan los planificadores del agua (UNESCO, 2012).

Los únicos métodos para aumentar el suministro de agua más allá del ciclo hidrológico son la desalinización y la reutilización del agua (Shannon et al., 2008). De éstos, la desalinización del agua de mar ofrece un suministro de agua de alta calidad, aparentemente ilimitado y constante, sin afectar a los ecosistemas naturales de agua dulce. La desalinización de las aguas subterráneas salobres es también una opción para aumentar el suministro de agua para las regiones continentales; sin embargo, la gestión de las salmueras de las plantas desalinizadoras continentales representa desafíos adicionales (Jenkins, Paduan, Roberts, Schlenk y Weis, 2012).

Las principales tecnologías de desalinización actualmente en uso se basan en la separación de membranas; por ejemplo, mediante ósmosis inversa (RO) yla destilación térmica (destilación por destello y destilación por efecto de varias etapas), y la $\mathrm{RO}$ representa más del 50\% de la capacidad instalada mundial (Zotalis et al., 2014; Zhou y Tol, 2005). 
Los procesos de desalinización térmica convencionales son ineficientes en el uso de energía y sufren particularmente de corrosión, así como de incrustaciones que también afectan a la osmosis inversa. Incluso cuando el combustible está disponible fácilmente y es de bajo costo, los altos costos operativos y de capital limitan la adopción. Por lo tanto, la cuota de mercado de las grandes desaladoras térmicas convencionales probablemente disminuirá (Shannon et al., 2008), (S. P. Chaurasia, Sushant Upadhyaya et al., 2011). En un futuro a medio plazo, la desalinización masiva de agua de mar mediante procesos térmicos podría lograrse utilizando la energía térmica almacenada en la corteza terrestre - la cual es suficiente para cubrir nuestras necesidades energéticas actuales durante aproximadamente 50 millones de años. La tecnología para cosechar esta enorme fuente de energía, por ejemplo, a través de sistemas geotérmicos mejorados (EGS) es técnicamente viable, pero aún necesita ser desarrollada (Laboratorio Nacional de Idaho, 2006).

Para familias y sistemas comunitarios pequeños en lugares remotos, especialmente en el mundo en desarrollo, la destilación solar de agua de mar, es decir, la generación de agua dulce con alambiques solares, tiene un gran potencial en las zonas costeras con acceso limitado a otras fuentes de energía que no sean la energía solar (Fath, 1998; Bourouni, Chaibi y Tadrist, 2001).

\section{Destilación solar de agua de mar}

La producción de agua potable alimentada por energía solar se ha utilizado desde tiempos antiguos. Los marineros fenicios (1500 a. C. -500 a. C.) que viajaron por el Mediterráneo ya utilizaban la radiación solar térmica para convertir el agua de mar en agua potable. El filósofo clásico griego Aristóteles describió el ciclo del agua en la naturaleza y señaló que cuando el agua salada se convierte en vapor y el vapor se condensa, lo hace en forma de agua dulce (Ross, LL. D. y Smith, 1931). Desde su introducción por los alquimistas árabes en 1551 (Ferrario, 2007) la tecnología de destilación se ha aplicado a la producción de agua dulce durante siglos. E1 número de los diferentes diseños solares existentes en la actualidad es abrumador (Dsilva Winfred Rufuss y otros, 2016), (Kalogirou, 2005). Uno de los principales inconvenientes de estos sistemas es su limitada capacidad de producción de agua dulce, que según la bibliografía muestra una capacidad de desalinización, generalmente inferior a 0,6 1 de agua dulce por hora $\mathrm{y}^{2}$ de superficie expuesta al sol, con una irradiancia de $1 \mathrm{KWh}^{-1}$ (Sivakumar y Ganapatía Sundaram, 2013).

Sin embargo, recientemente, los nuevos enfoques en materiales impulsados por el surgimiento y la proliferación de nanociencias y nanotecnologías han creado un enorme potencial de innovación, también para la mejora de la eficiencia de los alambiques solares. Como se muestra esquemáticamente en la Figura n. 2, las áreas de nanomejora para los alambiques solares son múltiples: los nanomateriales pueden optimizar notablemente el aislamiento térmico (reducción de pérdidas de calor (Roco, Mirkin y Hersam, 2010; Nikita Aigner, 2011), mejorar el pretratamiento y post-tratamiento del agua, optimizar la condensación del agua (Yoon et al, 2012; Chen et al., 2016) y mejorar la captación de luz solar con materiales ópticos avanzados (McClean-Ilten y Zerulla, 2016). 
Un gran avance en la investigación de la evaporación de agua inducida por luz con nanomateriales se ha logrado gracias al reciente descubrimiento de que la eficiencia de la evaporación puede ser significativamente mejorada mediante el uso de superficies plasmónicas de nano materiales.

En este trabajo, nos centramos en este último descubrimiento, desarrollando nuevos enfoques para la evaporación inducida por luz mejorada en capas de material húmedo térmicamente aisladas con propiedades de absorción de luz extraordinarias (Figura n. 2 ). Nuestro enfoque apunta a una de las principales razones de las limitaciones observadas en la capacidad de desalinización de los alambiques solares convencionales. (Ver Figura n. ${ }^{\circ}$ 2)

Sólo una pequeña fracción de la luz solar incidente se convierte en la energía translacional que las moléculas de agua necesitan para realizar el cambio de fase.

En los enfoques termodinámicos clásicos $(\mathrm{Ni}$ et al., 2016), la tasa de evaporación del agua por unidad de superficie generada por el flujo solar ambiental se calcula como (Eq. 1).

$$
m / A=q_{\text {solar }} / h_{f g}(\text { Eq. 1) }
$$

Donde $m$ es el flujo de masa o la tasa de evaporación del agua $\left[\mathrm{Kgs}^{-1}\right], A$ es la superficie del agua frente al sol[ $\left.\mathrm{m}^{2}\right]$, q_solar es la medida del flujo solar en $\left[\mathrm{Wm}^{-2}\right]$ y $h_{f g}$ es calor latente de evaporación del agua utilizada en la literatura generalizada (Datt, 2011), con un valor de 2.260 $\mathrm{MJkg}^{-1}$
Eq. 1 conduce a una tasa máxima de evaporación de agua de 4.4 X $10-4 \mathrm{kgm}^{-2} \mathrm{~s}^{-1}$, o $1.58 \mathrm{kgm}^{-}$ ${ }^{2} \mathrm{~h}^{-1}$, para una superficie de agua de $1 \mathrm{~m} 2$ que está expuesta a un flujo solar de $1000 \mathrm{Wm}^{-2}$ (1 sol=1000 $\mathrm{Wm}^{-2}$ ), para un sistema ideal sin pérdidas de calor y con una eficiencia de generación de vapor $\eta_{\text {thermal }}$ del 100\%. Donde $\eta_{\text {thermal }}$

$$
\eta^{\text {thermal }}=m h_{f g} q_{\text {solar }}^{-1} A^{-1}(\text { Eq. 2) }
$$

Sin embargo, en los sistemas reales se producen pérdidas de calor. La evaporación del agua es un proceso superficial y sólo las moléculas de agua en la interfaz agua-aire delgada pueden ser conducidas a la fase de vapor a través de su estado de energía cinética. El calentamiento del agua en regiones no-superficiales transfiere energía a moléculas de agua que no participan directamente en el proceso de evaporación, induciendo cambios en sus estados vibratorios y/o relajando fuerzas atractivas entre estas moléculas no superficiales, conocidas como bulk molecules.

Otras contribuciones de pérdida de calor incluyen las pérdidas por convección a la fase gaseosa, así como las pérdidas de radiación en las interfases gas/líquido y líquido/sólido (ver Figura n. ${ }^{\circ}$ 3). Estas pérdidas de calor reducen significativamente las eficiencias de generación de vapor solar para superficies de agua expuestas al sol, llevando a valores $\eta_{\text {thermal }}$ bajos (inferiores al 40\%) en flujos solares $q_{\text {solar }}=1000 \mathrm{Wm}^{-2}$ (Bae et al., 2015; Morciano et al., 2017). 


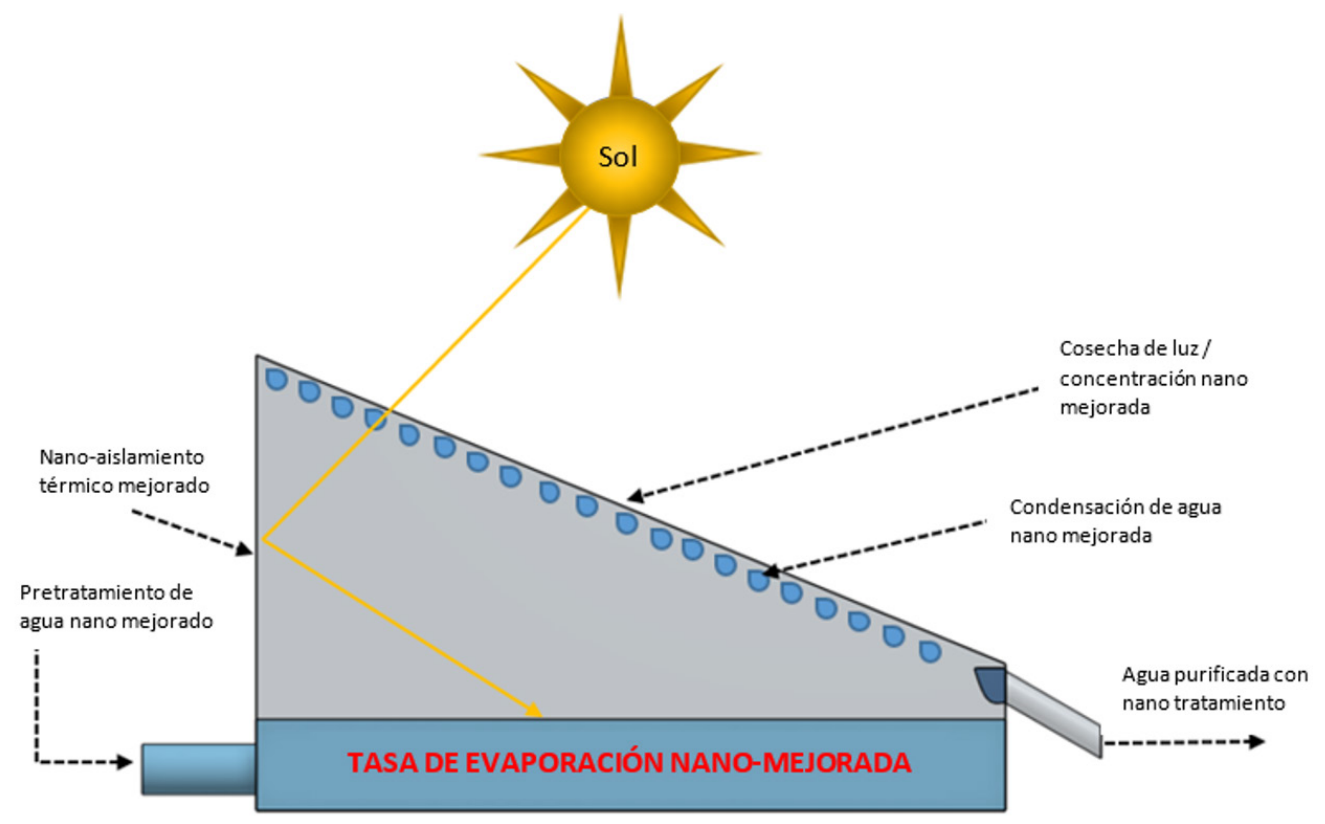

Figura $n .^{\circ}$ 2. Presentación esquemática del alambique solar convencional, mostrando las áreas potenciales para obtener aumentos de eficiencia mejorados en nanotecnología.

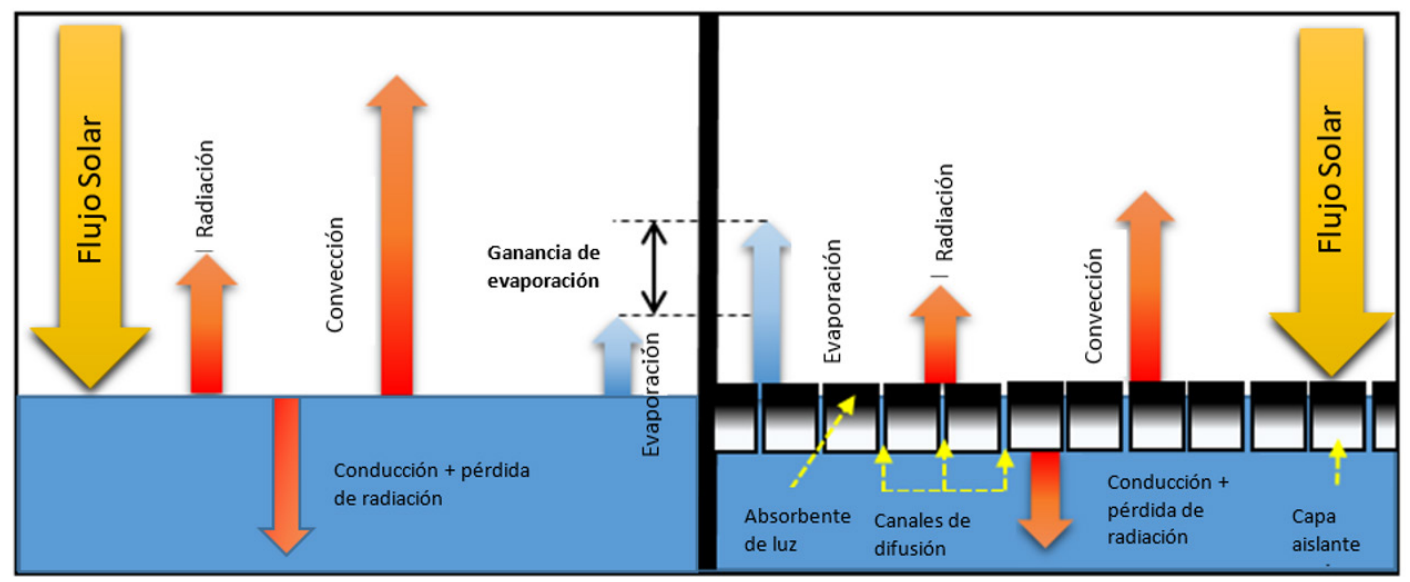

Figura n. ${ }^{\circ}$ 3. Disipación de energía solar de la superficie del agua irradiada. Izquierda: Pérdidas de energía de la superficie desnuda irradiada del agua. Derecha: Disipación de energía en la capa SLS impregnada de agua. La reducción de las pérdidas de calor conductivo y de radiación resulta en una notable ganancia de energía disponible para inducir cambios de fase, resultando en un aumento de $\eta_{\text {thermal }}$. Figura adaptada de (Ni et al., 2016) 
La reducción de las pérdidas de calor conductoras al agua en regiones no superficiales puede lograrse experimentalmente mediante el aislamiento de estas regiones de la radiación solar, por ejemplo, mediante estructuras flotantes de materiales porosos que absorben agua, como se muestra en la Figura n. ${ }^{\circ} 4$. Estas estructuras reducen significativamente el grosor de la capa de agua expuesta al sol y aíslan la masa de agua de las regiones de calientes en la superficie (Neumann et al., 2013).

Si el área expuesta al sol de la flotación está cubierta con materiales absorbentes de luz, la energía calorífica puede ser localizada cerca de la superficie del agua y puede inducir cambios de fase no equilibrados localizados de energía, generando vapor mientras que el medio circundante permanece frío (Boriskina et al., 2013). Como resultado, la eficiencia de la generación de vapor solar puede incrementar significativamente.

\section{Materiales y métodos}

Los experimentos de evaporación de agua de mar se realizaron en condiciones de laboratorio: $21^{\circ} \mathrm{C}$ regulados por aire acondicionado, 930 $\mathrm{hPa}$ de presión ambiental y $65 \%$ de humedad relativa. Un contenedor de agua impresa en 3D $(75 \times 75 \times 50 \mathrm{~mm})$ con una superficie cuadrada de $5,6 \times 10^{-3} \mathrm{~m}^{2}$ (Muñoz et al., 2016) fue llenado con $250 \mathrm{ml}$ de agua de mar obtenida de la costa del Océano Pacífico de El Salvador (Xanadú, La Libertad, con coordenadas geográficas $13^{\circ} 29^{\prime}$ 43.184"N 89 24' 30.556”O). Sobre la superficie del agua se colocó una estructura SLS flotante, compuesta por un flotador, material absorbente de agua (tejido de papel) y material finamente molido absorbente de luz (ver Figura n. ${ }^{\circ}$ 4) fue colocada sobre la superficie del agua.

La parte superior del SLS flotante; es decir, la superficie húmeda del material absorbente de luz, fue expuesta a la luz emitida por una fuente LED de 100W (Unbranded, Luminous Intensity: $8000-90001 \mathrm{~m}$, eBay), situada a $12 \mathrm{~cm}$ sobre la superficie de evaporación. (Ver Figura n. ${ }^{\circ}$ )

Las pérdidas de masa del depósito de agua se midieron con una báscula OHAUS con una precisión de $0.01 \mathrm{~g}$ (Figura n. ${ }^{\circ}$ 5). Las mediciones de la temperatura superficial de los materiales absorbentes de luz se realizaron con un termómetro infrarrojo portátil FLUKE 62 MAX.

La fuente de irradiación LED utilizada en los experimentos in situ tiene un sistema de enfriamiento activo, consistente en un disipador de calor soplado por un ventilador. Para evitar pérdidas de precisión en las mediciones de pérdida de peso, el experimento fue encerrado en una cámara oscura con aislamiento de viento y una abertura cuadrada cubierta de vidrio en la parte superior.

Con el fin de evitar gradientes de temperatura en la balanza inferior, la abertura se colocó a la distancia a la que la luz de la fuente LED se desplazó a través de la superficie de cristal e iluminó únicamente la superficie completa de la capa superior de la estructura sándwich flotante (Figura n. ${ }^{\circ}$ 6). (Ver Figura n. ${ }^{\circ}$ )

Todos los experimentos de evaporación se realizaron durante un período de tiempo de una hora, durante el cual se registraron la pérdida de peso y la temperatura a intervalos de 5 minutos. 


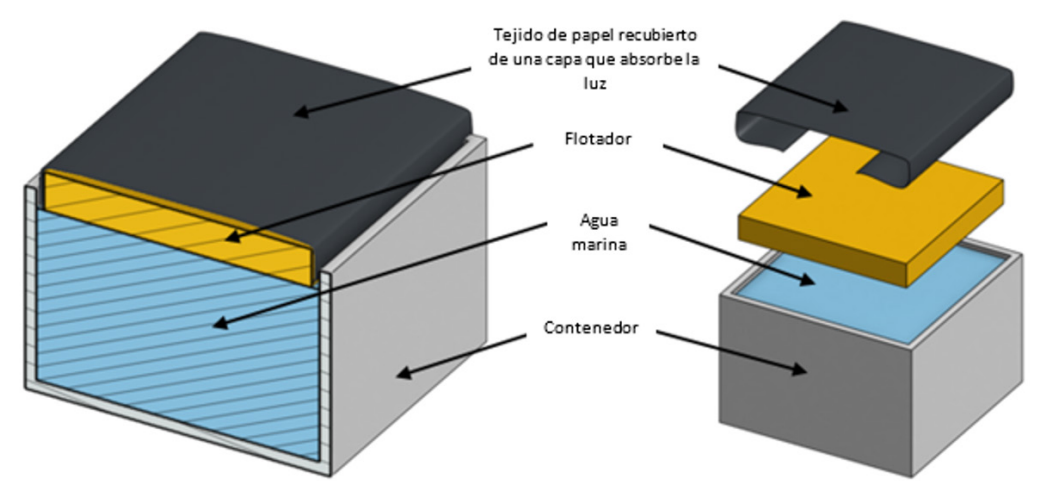

Figura $n .^{\circ} 4$. Vista esquemática de la celda de evaporación utilizada para los experimentos de evaporación. Izquierda: Sección de la celda de evaporación con estructura flotante Sandwich Layer Structure (SLS). El SLS consiste en un flotador de poliuretano recubierto con papel absorbente de agua (gris). La superficie superior del papel se cubre con finas capas de materiales absorbentes de la luz que se han investigado en este estudio. Derecha: Vista lateral SLS. El transporte de agua ocurre por acción capilar desde el contenedor hasta la superficie del SLS, humedeciendo el material absorbente de la luz y mejorando la evaporación.

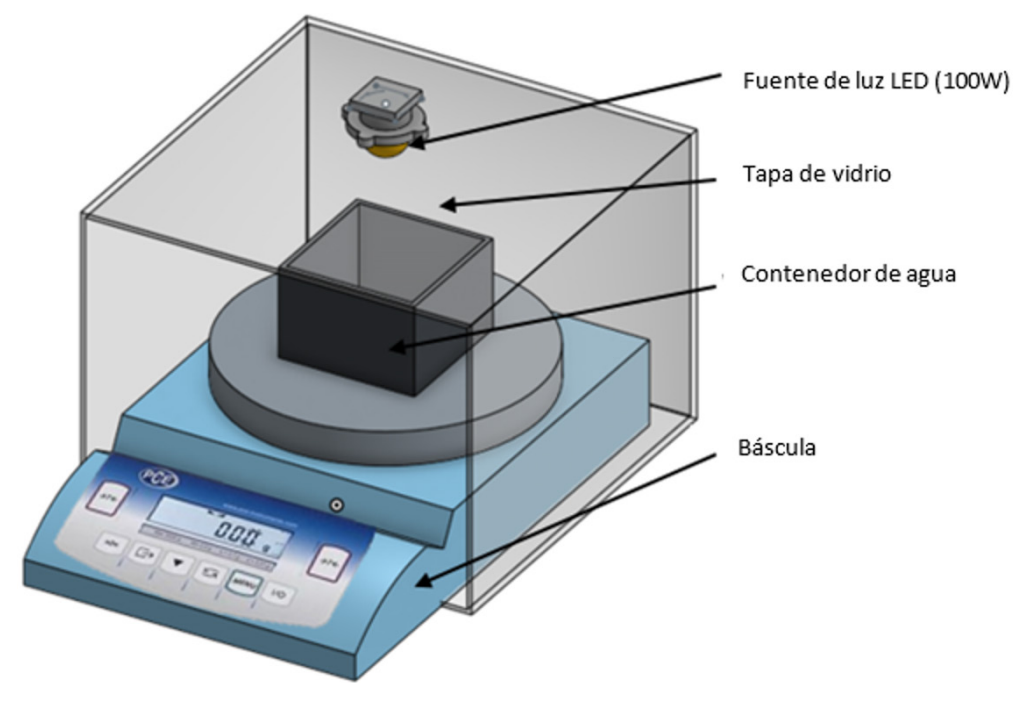

Figura $n .^{\circ}$. Vista esquemática de la configuración experimental para mediciones de pérdida de peso. El agua de mar en el contenedor se irradia con una fuente LED de 100W, causando evaporación y por lo tanto pérdida de masa del contenedor de agua. Los correspondientes transitorios de pérdida de peso del contenedor se registran con una báscula de precisión. La instalación incluye una cubierta, diseñada para evitar la iluminación de la báscula y proteger la superficie de evaporación de los flujos de aire convectivos generados por el sistema de aire acondicionado del laboratorio y el ventilador de refrigeración LED. 


\section{Resultados y discusión}

Estudios previos (Christoph et al., 2016) se demostró que la tasa de evaporación de las superficies de agua de mar directamente expuestas a la luz (sin SLS), medida bajo condiciones de temperatura, presión, humedad e intensidad de radiación prevalecientes descritas en la sección experimental, están en el orden de $0.126 \mathrm{Kgm}^{-2} \mathrm{~h}^{-1}$.

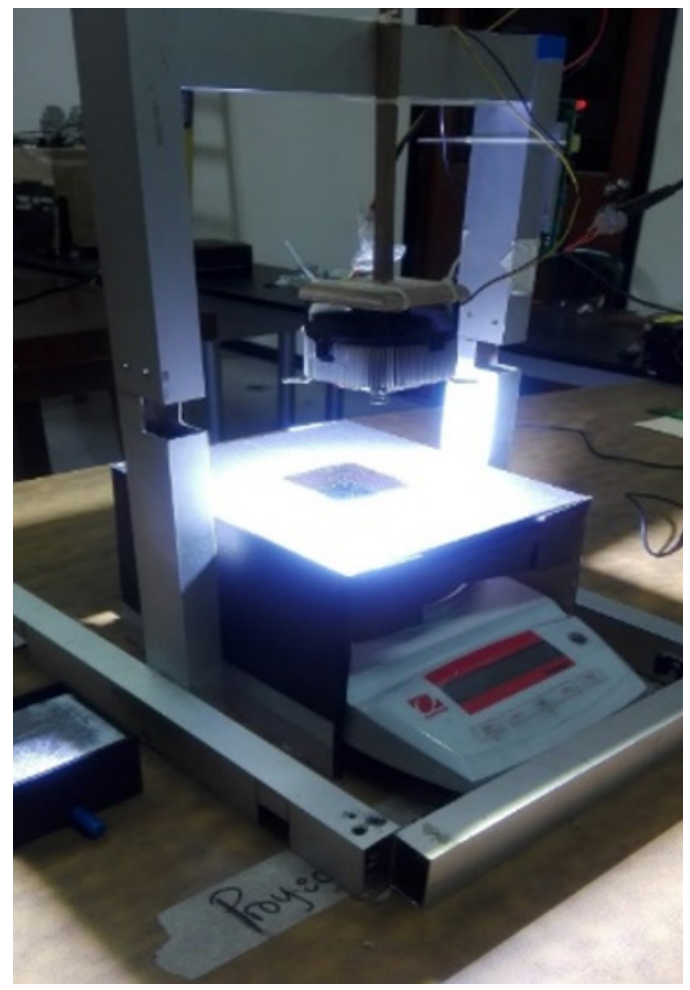

Figura n. ${ }^{\circ}$ 6. Experimento de evaporación de agua de mar, en su interior hay un contenedor de $75 \times 75 \times 50 \mathrm{~mm}$, el agua se irradia con una fuente de luz LED de 100W. La pérdida de peso inducida por la evaporación del agua y la temperatura superficial SLS fueron medidas a intervalos de 5 minutos durante 1 hora.
En un segundo conjunto de experimentos, realizados en condiciones experimentales idénticas, de superficies SLS impregnadas de agua desnuda, es decir, sin la presencia de capas de material absorbente de luz, se midió la pérdida de masa de agua por evaporación para diferentes tipos de tejidos absorbentes de agua a base de celulosa. (Ver Figura n. ${ }^{\circ}$ 7)

Los resultados de estos experimentos muestran claramente un aumento inducido por SLS de la pérdida de masa de agua, incluso en ausencia de capas de material absorbente de luz en la superficie expuesta a la luz. Este efecto se atribuye a la insulación térmica de las estructuras flotantes, que pierden al agua subterránea del recipiente. Se observó una evaporación significativamente mayor para el tejido de papel, demostrando la importante función del transporte de fluidos desde el bulto de agua subyacente a la superficie SLS para obtener eficiencias optimizadas de generación de vapor solar (Ito et al., 2015; L. Canbazoglu et al., 2016). (Ver Figura n. ${ }^{\circ} 7$ )

Un tercer conjunto de experimentos insitu se realizó con una capa superior de SLS consistente en diferentes materiales absorbentes de luz, incluyendo polvos de grafito usados en estudios previos (Christoph et al., 2016) y polvo magnético finamente molido extraído de rocas ígneas trituradas y arena de playa finamente molida (Costa del Sol, El Salvador, con coordenadas geográficas $13^{\circ} 20^{\prime} 37.021^{\prime} \mathrm{N}$ $89^{\circ}$ 0’ 23.003"O). Los diámetros de partículas de estos materiales, medidos por microscopía óptica, eran del orden de 5-10 micras. (Ver Figura n. ${ }^{\circ} 8$ ) 


\section{Comparación entre materiales absorbentes}

0.200

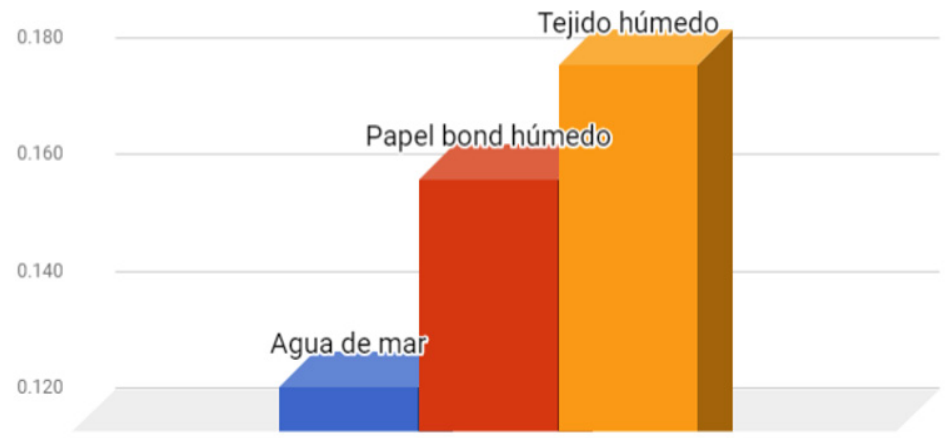

Figura $n .^{\circ} 7$. Las pérdidas de masa por evaporación del agua de mar (tasas de evaporación) expresadas en agua evaporada por metro cuadrado y hora, obtenidas, en las mismas condiciones experimentales para una superficie de agua desnuda (a), y en las superficies, dos materiales diferentes de absorción de agua de la capa SLS (b) y (c). Los resultados muestran que la introducción de una estructura flotante aislada conduce a una mejora significativa de la evaporación del agua, debido a la reducción de las pérdidas de calor al volumen de agua subyacente. Las diferentes tasas de evaporación del agua observadas para los distintos absorbedores de agua también indican la importancia del transporte de fluidos a través de la acción capilar desde el agua en el recipiente hasta la superficie del SLS.

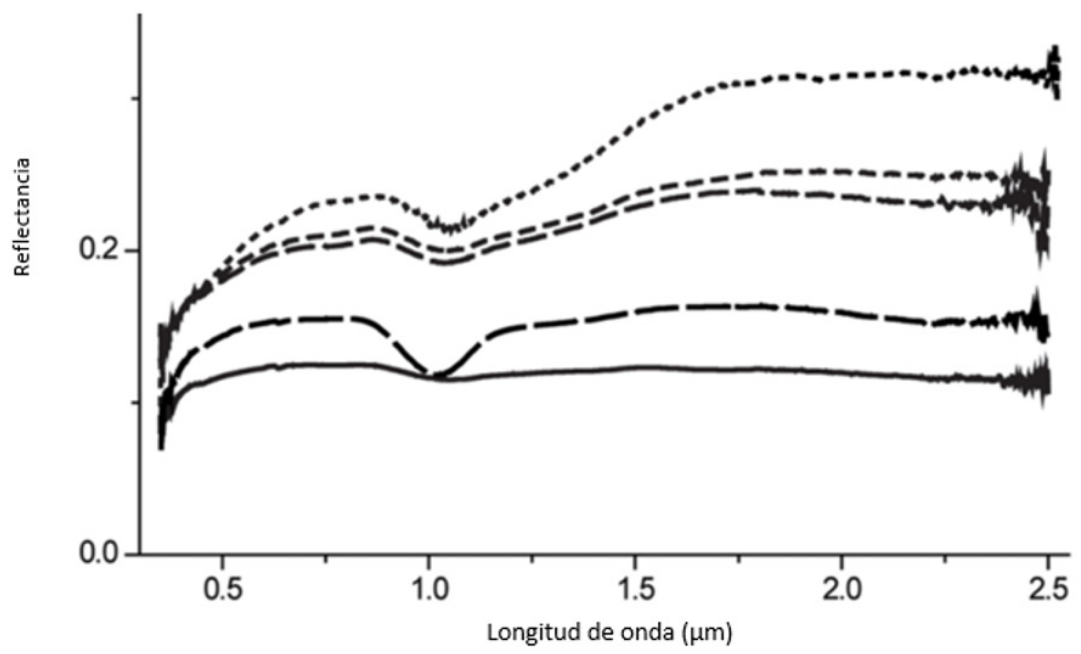

Figura $n .{ }^{\circ} 8$. Espectros de reflexión infrarroja de polvos de roca ígnea con composiciones de roca muy similares a granel (Carli, Serventi y Sgavetti, 2014) 
En la Figura n. 8 se muestran los resultados de estos experimentos. Reportamos los siguientes hallazgos:

- Las capas de grafito mejoran la evaporación del agua por un factor de dos a tres, en comparación con las superficies de agua no recubiertas. Estos resultados concuerdan con los valores obtenidos en estudios anteriores (Christoph et al., 2016).

- Las capas superiores SLS de los componentes de polvo de roca ígneo aumentan las tasas de evaporación del agua en un 120\%, en comparación con las tasas obtenidas con los polvos de grafito. Atribuimos este efecto a la banda de absorción IR más amplia del polvo magnético usado que se muestra en la Figura n. ${ }^{\circ} 8$.

- Observamos una alta correlación (R2=0.9481 y $\mathrm{p}=0.05$ ) entre las tasas de evaporación medidas y el diferencial de temperatura correspondiente de la superficie SLS irradiada. Altas temperaturas superficiales se traducen en altas tasas de evaporación. Esta observación es coherente con consideraciones termodinámicas básicas, es decir, el hecho de que, a una temperatura creciente, un mayor número de moléculas de agua tienen suficiente energía cinética para superar las fuerzas de atracción que prevalecen en la fase líquida. Otras explicaciones de nuestra observación con partículas magnéticas, como la presencia de puntos calientes localizados inducidos por los plasmones de superficie, requieren una confirmación experimental adicional.

- Para el caso de las partículas magnéticas, también observamos que el tiempo necesario para alcanzar la temperatura máxima de la superficie irradiada es notablemente menor que para el caso de los materiales grafitos.

\section{Ver Figura n. ${ }^{\circ} 9$}

La Figura n. 10 muestra el resultado de un cuarto conjunto de experimentos in situ, en los que se realizaron mediciones de evaporación a diferentes concentraciones de luz, variando las distancias de la lámpara LED en 12 y $6 \mathrm{~cm}$ con respecto a la superficie superior del SLS para comparar la pérdida de masa. Asumiendo que la lámpara se encuentra a una distancia promedio entre los valores anteriormente descritos es de aproximadamente 4 veces el incremento, el correspondiente aumento de la pérdida de masa debe ser al menos el mismo, asumiendo que $\eta_{\text {thermal }}$ permanece inalterado. Sin embargo, nuestros resultados muestran que, con una intensidad de luz doble, la pérdida de masa aumenta sólo en un factor de 2.0 (componentes de agua y polvo de roca ígnea) y en un factor de 2.7 para el caso del polvo de grafito.

Los resultados de otros autores (Boriskina et al., 2013; Wen Shang et al., 2014), obtenidos en materiales plasmónicos irradiados, incluyendo nanopartículas de oro, plata y grafeno, reportan un aumento de $\eta_{\text {thermal }}$ con una concentración lumínica creciente, lo que se atribuye a la presencia de plasmones superficiales. Nuestros resultados, obtenidos con granos en polvo de tamaño micrón no indican tal hallazgo, sugiriendo que no hay actividad plasmónica en las partículas de los materiales utilizados. Queda por confirmar si partículas de menor tamaño muestran dicho efecto. 

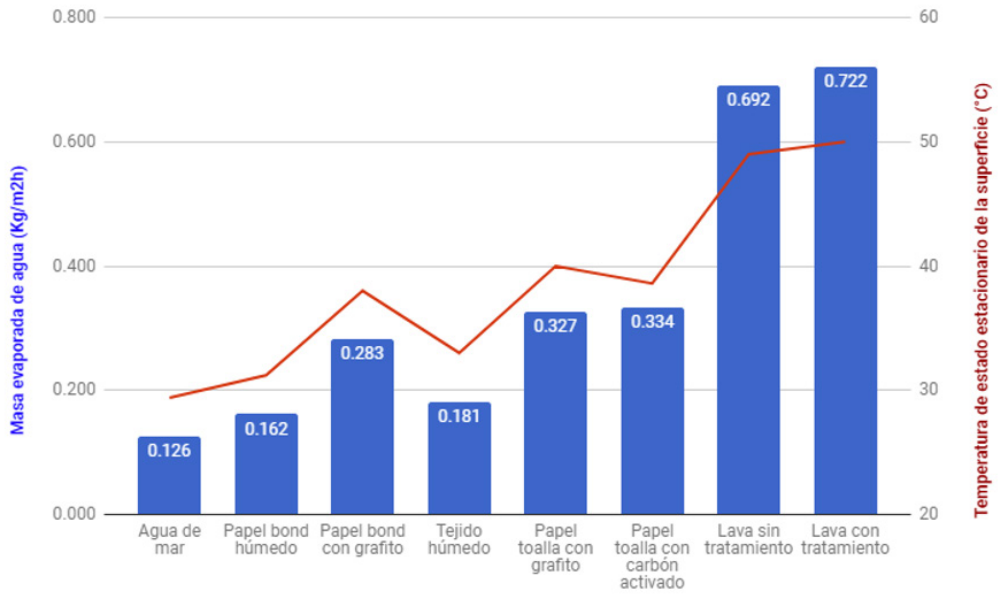

Figura n. $^{\circ}$ 9. Gráfico comparativo de las velocidades de evaporación y temperaturas máximas de superficie SLS obtenidas con diferentes capas superiores SLS absorbentes de luz. Los datos muestran una buena correlación entre la tasa de evaporación medida y las temperaturas máximas de superficie de la capa superior alcanzadas durante 1 hora de irradiación.

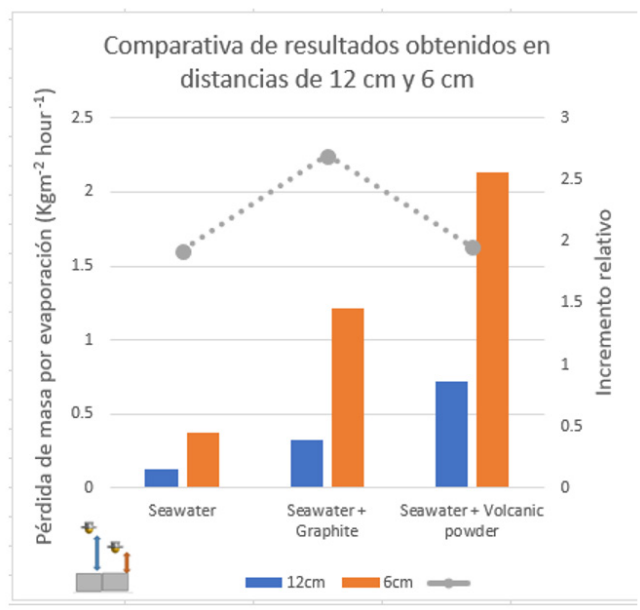

Figura $n .{ }^{\circ}$ 10. Resultados comparativos de los experimentos de evaporación del agua, en los que se midieron tasas de pérdida de masa a diferentes intensidades de luz en superficies de agua de mar descubiertas, y en superficies SLS con componentes de grafito y polvo de roca ígnea. El aumento relativo de la pérdida de masa es mayor en el caso del grafito, mientras que los componentes de polvo de roca ígneo muestran que la mayor tasa de pérdida de masa se midió para los componentes de polvo de roca ígnea. 


\section{Conclusiones}

La evaporación de agua inducida por luz se puede incrementar significativamente cuando se utilizan capas de polvo húmedo térmicamente insufladas.

Los experimentos in situ realizados con estructuras SLS flotantes, recubiertas con polvo de roca ígnea, duplican la tasa de evaporación medida respecto a superficies con polvo de grafito. Se observó un aumento quíntuple de los valores correspondientes obtenidos con agua donde tanto su superficie, como su interior son expuestos a la irradiación.

Durante la fase de pruebas se aumentaron las concentraciones de luz, pero no se observó incremento $\eta_{\text {thermal }}$ sin embargo, esto podría explicarse debido a que las partículas oscilaban en tamaños medios y grandes (80 a $240 \mu \mathrm{m}$ ), se necesitan pruebas experimentales para apoyar esta explicación.

Para tener una mayor comprensión acerca de la evaporación del agua en proximidad de superficies irradiadas es necesario entender diversos procesos entre los cuales cabe mencionar; la transferencia de calor entre las nanopartículas y las fases vapor/ líquido del agua en escala nanométrica (es decir difusivo o balístico), el mecanismo de nucleación de la fase de vapor, su influencia del campo de presión en la transferencia de calor, la cinética de crecimiento de la fase de vapor, la flotabilidad de partículas inducida por vapor, las fluctuaciones de energía absorbida debido a la formación de fase de vapor y la radiación entre las fases.

Como siguiente paso, se realizarán experimentos cuantitativos en condiciones in vivo. Destiladores solares mejorados SLS con áreas de captura de luz en el orden del metro cuadrado están planificados para ser colocados en las regiones costeras de El Salvador y monitoreados vía tecnología IoT.

\section{Bibliografía}

Bae, K. et al. (2015) Flexible thin-film black gold membranes with ultrabroadband plasmonic nanofocusing for efficient solar vapour generation.', Nature communications. Nature Publishing Group, 6, p. 10103. doi: 10.1038/ncomms10103

Bates, B. C. et al. (2008) Climate Change and Water, Climate change and water. doi: 10.1016/j. jmb.2010.08.039.

Boriskina, S. V., Ghasemi, H. and Chen, G. (2013) 'Plasmonic materials for energy: From physics to applications', Materials Today. Elsevier Ltd., 16(10), pp. 375-386. doi: 10.1016/j. mattod.2013.09.003.

Bourouni, K., Chaibi, M. T. and Tadrist, L. (2001) 'Water desalination by humidification and dehumidification of air: State of the art', Desalination, 137(1-3), pp. 167-176. doi: 10.1016/S0011-9164(01)00215-6.

Canbazoglu, F. M. et al. (2016) 'Enhanced solar evaporation of water from porous media, through capillary mediated forces and surface treatment', AIP Advances, 6(8), pp. 1-16. doi: 10.1063/1.4961945.

Carli, C., Serventi, G. and Sgavetti, M. (2014) 'VNIR spectral characteristics of terrestrial igneous effusive rocks: mineralogical composition 
and the influence of texture', Geological Society, London, Special Publications, 401, pp. 139-158. doi: 10.1144/SP401.19.

Chen, X. et al. (2016) 'Coalescence-Induced Jumping of Multiple Condensate Droplets on Hierarchical Superhydrophobic Surfaces', Scientific Reports. American Chemical Society, 6(1), p. 18649. doi: 10.1038/srep18649.

Christoph, R. et al. (2016) 'Estudios de materiales grafíticos comunes como aceleradores de evaporación de agua bajo irradiación luminosa', Boletin Nanotecnia, September. Available at: http://nanotecnialab.ufg.edu.sv/frm/newsletters/ Nanoboletin_Septiembre-2016.pdf (Accessed: 16 November 2017).

Datt, P. (2011) 'Latent Heat of Vaporization/ Condensation', in. Springer Netherlands, pp. 703703. doi: 10.1007/978-90-481-2642-2_327.

Dsilva Winfred Rufuss, D. et al. (2016) 'Solar stills: A comprehensive review of designs, performance and material advances', Renewable and Sustainable Energy Reviews, 63, pp. 464496. doi: 10.1016/j.rser.2016.05.068.

Fath, H. E. S. (1998) 'Solar distillation: a promising alternative for water provision with free energy, simple technology and a clean environment', Desalination. Elsevier, 116(1), pp. 45-56. doi: 10.1016/S0011-9164(98)00056-3.

Ferrario, Ga. (2007) Al-Kimiya: Notes on Arabic Alchemy. Available at: https://www. chemheritage.org/distillations/magazine/alkimiya-notes-on-arabic-alchemy (Accessed: 5 October 2017).
Gleick, P. H. (1993) Water in crisis a guide to the world's fresh water resources, Water in crisis a guide to the world's fresh water resources. Oxford University Press. doi: 10.2307/2623756.

Haddeland, I. et al. (2014) 'Global water resources affected by human interventions and climate change (SI)', Proceedings of the National Academy of Sciences, 111(9), pp. 3251-3256. doi: 10.1073/pnas.1222475110.

Idaho National Laboratory (2006) The Future of Geothermal Energy, The Future of Geothermal Energy. Disponible en: http:// www.eere.energy.gov/geothermal/pdfs/ structure_outcome.pdf.

Ito, Y. et al. (2015) 'Multifunctional Porous Graphene for High-Efficiency Steam Generation by Heat Localization', Advanced Materials, 27(29), pp. 4302-4307. doi: 10.1002/ adma.201501832.

Kalogirou, S. A. (2005) 'Seawater desalination using renewable energy sources', Progress in Energy and Combustion Science, 31(3), pp. 242281. doi: 10.1016/j.pecs.2005.03.001.

McClean-Ilten, É. and Zerulla, D. (2016) 'Nanoscale Tailored Plasmonic Material for Optimum Broadband Solar Harvesting', Advanced Optical Materials, 4(3), pp. 413-418. doi: 10.1002/adom.201500513.

Morciano, M. et al. (2017) 'Efficient steam generation by inexpensive narrow gap evaporation device for solar applications', Scientific Reports, (February), pp. 1-9. doi: 10.1038/s41598-01712152-6. 
Muñoz, R. et al. (2016) 'Prototipado rápido de componentes para sistema de estudio de desalinización solar mejorada', Nanotecnia: Boletín Mensual Divulgativo, (Octubre). Disponible en: http://nanotecnialab.ufg.edu.sv/frm/newsletters/ Nanoboletin_Octubre-2016.pdf (Accessed: 16 November 2017).

NASA Climate Change Webpage (2017) (no date). Disponible en: https://climate.nasa.gov/.

Neumann, O. etal. (2013) 'Solar vapor generation enabled by nanoparticles', ACS Nano, 7(1), pp. 42-49. doi: 10.1021/nn304948h.

Ni, G. et al. (2016) 'Steam generation under one sun enabled by a floating structure with thermal concentration', Nature Energy, 1(9), p. 16126. doi: 10.1038/nenergy.2016.126.

Nikita Aigner (2011) Bacterial cellulose: not just a lightweight food, but also a fascinating scaffolding material. Universität für Bodenkultur, Wien.

Roco, M. C., Mirkin, C. A. and Hersam, M. C. (2010) 'Nanotechnology research directions for societal needs in 2020 - Retrospective and Outlook', WTEC-World Technology Evaluation Center, pp. 476-477. doi: 10.1007/978-94-007-1168-6.

Ross, W. D., LL.D. and Smith, J. A. (1931) Aristotele's METEOROLOGICA translated.

S. P. Chaurasia, Sushant Upadhyaya, K. S. et al. (2011) 'Seawater Desalination: Trends and Technologies', Desalation, Trends and Technologies, 50(Md), p. 334. doi: 10.5772/60142.
Service, R. F. (2006) 'Desalination Freshens Up', Science. American Association for the Advancement of Science, 313(5790), pp. 1088-1090. doi: 10.1126/science.313.5790.1088.

Shannon, M. A. et al. (2008) 'Science and technology for water purification in the coming decades', Nature, 452(7185), pp. 301-310. doi: $10.1038 /$ nature06599.

Sivakumar, V. and Ganapathy Sundaram, E. (2013) 'Improvement techniques of solar still efficiency: A review', Renewable and Sustainable Energy Reviews. Elsevier, 28(July), pp. 246-264. doi: 10.1016/j.rser.2013.07.037.

Supply, F. et al. (2011) 'GEMI Water Sustainability Tool'. Available at: http://gemi.org/water/ watertrends.htm (Accessed: 5 October 2017).

UN-WWAP (2015) The United Nations World Water Development Report 2015: Water for a Sustainable World. doi: 978-92-3-100071-3.

UNESCO (2012) World Water Development Report Volume 4: Managing Water under Uncertainty and Risk, UN Water Report. doi: 10.1608/FRJ-3.1.2.

Villanueva, L. G., Bausells, J. and Brugger, J. (2016) 'Grand Challenge in N/MEMS', Frontiers in Mechanical Engineering, 1(JANUARY). doi: 10.3389/fmech.2015.00015.

Yoon, D. et al. (2012) 'Enhanced condensation, agglomeration, and rejection of water vapor by superhydrophobic aligned multiwalled carbon nanotube membranes', ACS Nano, 6(7), pp. 5980-5987. doi: 10.1021/nn3008756. 
Zhou, Y. and Tol, R. S. J. (2005) 'Evaluating the costs of desalination and water transport', Water Resources Research, 41(3), pp. 1-10. doi: 10.1029/2004WR003749.

Zotalis, K.etal.(2014)'Desalination technologies: Hellenic experience', Water (Switzerland), 6(5), pp. 1134-1150. doi: 10.3390/w6051134

Jenkins, Paduan, Roberts, Schlenk y Weis. (2012). 'Management of Brine Discharges to Coastal Waters Recommendations of a Science Advisory Panel' Recuperado de: ftp://ftp. sccwrp.org/pub/download/DOCUMENTS/ TechnicalReports/694_BrinePanelReport.pdf
Zhenhui Wang, Yanming Liu, Peng Tao, Qingchen Shen, Nan Yi, Fangyu Zhang, Quanlong Liu, Chengyi Song, Di Zhang. (2014). 'Bio-Inspired Evaporation Through Plasmonic Film of Nanoparticles at the AirWater Interface'. Nano.Micro Small. https://doi. org/10.1002/smll.201401071 